\title{
A NOTE ON THE COMPONENT LIFETIME ESTIMATION OF A MULTISTATE MONOTONE SYSTEM THROUGH THE SYSTEM LIFETIME
}

\author{
VANDERLEI COSTA BUENO, Universidade de São Paulo
}

\begin{abstract}
In this paper we consider the observed lifetime of a multistate monotone system and 'the critical lower set' which causes the system deterioration. Then, under suitable conditions, we identify the component lifetime distribution using a Newton-Kantorovic iterative procedure as in Meilijson (1981).
\end{abstract}

CRITICAL LOWER SET; DETERIORATING SYSTEM

\section{Introduction}

Given the joint distribution of the lifetime $T$ of a binary coherent system and the set $K$ of components which cause the death of the machine, Meilijson (1981) identifies the component life distributions, which are assumed to be non-atomic and possess the same essential extrema. He uses a Newton-Kantorovic iterative method, under an assumption of the rank of the incidence matrix.

In this paper we propose to study the same problem for a multistate monotone system.

\section{Preliminaries and notation}

First we consider the stochastic behavior of the system and its components. Let $\left\{X_{i}(t)\right.$, $t \geqq 0\}$ be a right-continuous non-increasing stochastic process with values in $S=$ $\{0,1, \cdots, m\}$, representing the statistical behavior of component $i, i=1, \cdots, n$. The multistate monotone system is represented by $\{\phi(X(t)), t \geqq 0\}$ where $\phi: S^{n} \rightarrow S$ is a non-decreasing function and $X(t)=\left(X_{1}(t), \cdots, X_{n}(t)\right)$. Also assume that $\phi(0, \cdots, 0)=0$ and $\phi(m, \ldots, m)=m$.

Block and Savits (1982) decomposed $\phi(X(t))$ into binary structures:

$$
\psi_{k}(\beta(X(t)))=\min _{z \in L_{k}} \max _{(i, j) \in L_{k}(z)} \beta_{i j}(X(t)), \quad k=0, \cdots, m-1,
$$

where $\beta(\boldsymbol{X}(t))$ is the vector $\left(\beta_{i j}(\boldsymbol{X}(t)), 1 \leqq i \leqq n, 0 \leqq j \leqq m-1\right)$ lexicographically ordered and $\beta_{i j}(X(t))=1$ if $X_{i}(t)>j$ and 0 otherwise. $L_{k}$ is the set of all critical lower vectors for level $k$ and $L_{k}(z)=\left\{\left(i, z_{i}\right): z_{i} \neq m\right\}$.

The binary structure $\psi_{k}(\beta(\boldsymbol{X}(t)))$ characterizes the level $k$ of the multistate monotone system $\phi$ in the sense that $\phi(X(t)) \leqq k$ if and only if $\psi_{k}(\beta(X(t)))=0$.

We define $T_{i j}=\inf \left\{t \geqq 0: X_{i}(t) \leqq j\right\} ; i=1, \cdots, n ; j=0, \cdots, m-1$ and $T_{k}=\inf \{t \geqq 0$ : $\phi(X(t)) \leqq k\}, k=0, \cdots, m-1$. It is easy to prove that

$$
T_{k}=\inf \left\{t \geqq 0: \psi_{k}(\beta(X(t)))=0\right\}=\min _{z \in L_{k}} \max _{(i, j) \in L_{k}(z)} T_{i j} .
$$

Received 30 October 1987; revision received 6 May 1988.

Postal Address: Instituto de Matemática e Estatística da Universidade de São Paulo, Caixa Postal 20570 (Ag. Iguatemi), São Paulo, Brasil.

Supported in part by CNPq-Brasil. 
That means, to observe the first time that the system deteriorates to at most level $k$ is the same as observing the lifetime of the binary system $\psi_{k}(\beta(X(t)))$.

\section{The main result}

In our interpretation the observer allows the system to deteriorate until reaching the next level lower than $k+1$ and then makes an autopsy which reveals the 'critical lower set' that caused the allowed deterioration.

Let $F_{i j}(\cdot)$ be the distribution function of $T_{i j}, i=1, \cdots, n ; j=0, \cdots, m-1$ and let $M$ be the incidence matrix of order $\sum_{k=0}^{m-1} \#\left(L_{k}\right) \times m n$ whose rows are determined by $L_{k}(z), z \in L_{k}$, $k=0, \cdots, m-1$, in the following manner:

$$
M_{i j}=1 \quad \text { if } \quad(i, j) \in L_{k}(z) \text { and } M_{i j}=0 \quad \text { if } \quad(i, j) \notin L_{k}(z) .
$$

We assume that:

(i) The rank of $M$ is $m n$;

(ii) All $F_{i j}$ 's are non-atomic and possess the same essential infimum and the same essential supremum.

Under these hypotheses we can consider the identification problem. After the observation and diagnosis of $T_{k}$ suppose that we have

$$
\left\{T_{k}=t, L_{k}(z)\right\}=\left\{\max _{(i, j) \notin L_{k}(z)} T_{i j}=t, \min _{(i, j) \in L_{k}(z)} T_{i j}>t\right\} .
$$

Then we can calculate the distribution function:

$$
G_{L_{k}(z)}(x)=P\left\{T_{k} \leqq x, L_{k}(z)\right\}=\int_{0}^{x} \prod_{(i, j) \notin L_{k}(z)}\left(1-F_{i j}(t)\right) d\left[\prod_{(i, j) \in L_{k}(z)} F_{i j}(t)\right] .
$$

Differentiating (Radon-Nikodym) both sides and dividing by the integrand and then integrating, we obtain:

$$
\prod_{(i, j) \in L_{k}(z)} F_{i j}(x)=\int_{0}^{x}\left[\prod_{(i, j) \notin L_{k}(z)}\left(1-F_{i j}(t)\right)\right]^{-1} d G_{L_{k}(z)}(t) .
$$

It is important to note that for both of the above products and for each $i, i=1, \cdots, n$, there is only one $j$ such that the pair $(i, j)$ is a term of the product. With this interpretation, we define the matrix $\bar{M}=\left[\bar{M}_{i j}\right]$ of order $\sum_{k=0}^{m-1} \#\left(L_{k}\right) \times m n$, where each row corresponds to a critical lower set $L_{k}(z)$. For this row $\bar{M}_{i j}=1$ if $(i, j)$ is a term of the product $\Pi_{(i, j) \notin L_{k}(z)}(1-$ $\left.F_{i j}(t)\right)^{-1}$ and $\bar{M}_{i j}=0$, otherwise.

Furthermore, we denote:

$$
F(\cdot)=\left(F_{10}(\cdot), \cdots, F_{1 m-1}(\cdot), F_{20}(\cdot), \cdots, F_{2 m-1}(\cdot), \cdots, F_{n m-1}(\cdot)\right)^{t}
$$

and

$$
G(\cdot)=\left(G_{L_{k}(z)}(\cdot), z \in L_{k}, k=0, \cdots, m-1\right)^{t}, \quad \text { lexicographically ordered. }
$$

Also we consider that, if $h$ is a function of one variable and $\boldsymbol{V}$ is a vector, $h(\boldsymbol{V})$ is the vector whose $i j$ th coordinate is $h\left(V_{i j}\right)$ and the product of two column vectors is their coordinatewise multiplication.

With these considerations the logarithm of the equation (1) is the row of the matrix equation corresponding to $L_{k}(z)$.

$$
M \log F(x)=\log \int_{0}^{x} \exp \{-\bar{M} \log (1-F(t))\} d G(t) .
$$

Now, under the assumption that the rank of $M$ is $m n$, we can calculate $T=\left(M^{t} M\right)^{-1} M^{t}$ to 
obtain

$$
F(x)=\exp \left\{T \log \int_{0}^{x} \exp \{-\bar{M} \log (1-F(t))\} d G(t)\right\}
$$

which is an implicit equation for $F$.

Meilijson (1981) put this equation in the form:

$$
\exp \{-\boldsymbol{V}(x)\}+\exp \left\{T \log \int_{0}^{x} \exp \{\bar{M} V(t)\} d G(t)\right\}-1=0
$$

where $V_{i j}(x)=-\log \left(1-F_{i j}(x)\right)$, and considers $V$ as an element of the Banach space $B\left[0, x_{0}\right]$ of $R^{n m}$-valued, Borel-measurable bounded functions on $\left[0, x_{0}\right]$ with the supremum norm $\sup _{x} \max \left|V_{i j}(x)\right|$. He shows, using a Newton-Kantorovic iterative method, that this equation possesses a unique root. This root is the vector of lifetime distribution of the components.

\section{Examples}

We are going to use Barlow and Wu's (1978) structure function to produce an example. It is easy to show that for this structure,

$$
L_{k}=\left\{(m-k) \boldsymbol{x}+\boldsymbol{k}: \boldsymbol{x} \in K_{0}\right\}
$$

and

$$
L_{k}((m-k) \boldsymbol{x}+\boldsymbol{k})=\left\{(i, k): i \in K_{0}(\boldsymbol{x})\right\}
$$

where $K_{0}$ is the set of all minimal cut vectors of a binary coherent system (as in Barlow and Proschan (1981)) and $K_{0}(\boldsymbol{x})$ is the minimal cut set corresponding to the minimal cut vector $\boldsymbol{x}$.

Now let us consider the $(n-r+1)$ out-of- $n$ system. The system fails if and only if $r$ of the $r$ components fail. So $\#\left(L_{k}\right)=\left(\begin{array}{l}n \\ r\end{array}\right)$ and $\sum_{k=0}^{m-1} \#\left(L_{k}\right)=m\left(\begin{array}{l}n \\ r\end{array}\right)$. Furthermore, each row of the incidence matrix $M$ is a $m n$ vector with $r 1$ 's and $(m n-r) 0$ 's. So, a diagonal element of $M^{t} M$ is equal to $m\left(\begin{array}{c}m n-1 \\ r-1\end{array}\right)$ and all of the off-diagonal terms equal $m\left(\begin{array}{c}m n-2 \\ r-2\end{array}\right)$. We conclude that $M^{t} M$ is non-singular and that the implicit equations for $F$ become

where

$$
F_{i j}(x)=\left(\frac{\prod_{L_{k}(z):(i, j) \in L_{k}(z)} H_{L_{k}(z)}}{\left[\prod_{L_{k}(z):(i, j) \notin L_{k}(z)} H_{L_{k}(z)}\right] \frac{r(r-1)}{(r(m n+1-r)-1)}}\right) \frac{1}{m\left(\begin{array}{c}
m n-1 \\
r-1
\end{array}\right)}
$$

$$
H_{L_{k}(z)}=\int_{0}^{x}\left[\prod_{(i, j) \notin L_{k}(z)}\left(1-F_{i j}(t)\right)\right]^{-1} d G_{L_{k}(z)}(t) .
$$

\section{Acknowledgement}

I thank the editor and the referee for various comments and suggestions on a previous draft of this letter.

\section{References}

Barlow, R. E. AND Proschan, F. (1981) Statistical Theory of Reliability and Life Testing: Probability Models. To begin with; Silver Spring, MD. 
Barlow, R. E. AND Wu, A. S. (1978) Coherent systems with multistate components. Math. Operat. Res. 3, 275-281.

Block, H. W. AND Savits, T. H. (1982) A decomposition for multistate monotone systems. J. Appl. Prob. 19, 391-402.

MeIlisson, I. (1981) Estimation of the lifetime distribution of the parts from the autopsy statistics of the machine. J. Appl. Prob. 18, 829-838. 